Francis G. King MD FRCPC,

Amin M. Addetia MB ChB FRCSC,

Sharon D. Peters MD FRCPC, Gregory O. Peachey MD

\title{
Prophylactic lidocaine for postoperative coronary artery bypass patients, a double- blind, randomized trial
}

This double-blind controlled study examined the frequency of ventricular arrhythmias (premature ventricular contractions $(P V C s)>5 \cdot \mathrm{min}^{-1}$, bigeminy, couplets, ventricular tachycardia, and ventricular fibrillation) in coronary artery bypass graft (CABG) patients during the first $24 \mathrm{hr}$ postoperatively to determine the effect of prophylactic lidocaine on reducing the frequency of ventricular arrhythmias. Patients were included in the study if they had undergone CABG only, and had not received treatment for ventricular arrhythmias before coming off cardiopulmonary bypass. A total of 83 patients were studied and were randomly allocated to 43 in the placebo control group and 40 in the lidocaine-treated group. The results showed that 67 per cent of patients in the placebo group and 33 per cent of patients in the lidocaine treated group had ventricular arrhythmias $(P<0.005)$. There was also a significant reduction in ventricular fibrillation and ventricular tachycardia in the lidocaine treated group $(P<0.01)$. It is recommended that a routine infusion of lidocaine, $100 \mathrm{mg}$ bolus followed by 2 $m g \cdot \mathrm{kg}^{-1}$, be given to every postoperative coronary artery bypass patient for at least the first 24 hours.

Pendant les 24 premières heures suivant une revascularisation coronarienne, nous avons mesuré dans une étude randomisée à double insu, l'effet d' une infusion prophylactique de lidocaine

\section{Key words}

ANAESTHESIA: cardiac; COMPLICATIONS: arrhythmias;

HEART: arrhythmia, lidocaine, prevention, ventricular; SURGERY: cardiac.

From the Departments of Anaesthcsia, Intensive Care, and Cardiovascular Surgery, Faculty of Medicine, Memorial University of Newfoundland.

Address correspondence to: Dr. F.G. King, Discipline of Anaesthesia, Health Sciences Centre, St. John's, Newfoundland, Canada A1B 3V6. sur la fréquence des arythmies ventriculaires (extrasystoles ventriculaires $>5 \mathrm{~min}^{-1}$, bigéminisme, doublets, tachycardic et fibrillation ventriculaires). N'étaient inclus dans l'érude que les patients ayant subi une revascularisation seule et n'ayant eu aucune arythmie ventriculaire nécessitant une traitement avam le sevrage de la circulation extra-corporelle. 11 appert que 67 pour cent des 43 patients du groupe placébo et 33 pour cent des 40 patients du groupe lidocaine eurent des arythmies ventriculaires $(P<0,005)$. La tachycardie et la fibrillation ventriculaires étaient aussi moins fréquentes dans le groupe lidocaîne $(P$ $<0,01)$. Nous recommandons, dans tous les cas de revascularisation coronarienne, l'utilisation prophylactique de lidocaine à raison d'un bolus de $100 \mathrm{mg}$ suivi d'une perfusion à $2 \mathrm{mg}$ min $^{-1}$ pendant au moins 24 heures.

A number of studies has shown an increase in the likelihood of death as ventricular extrasystoles increase in the presence of coronary heart disease. ${ }^{1-3}$ Patients with coronary heart disease who undergo coronary artery bypass grafts (CABG) are also at increased risk for ventricular arrhythmias. Intraoperative and postoperative Holter monitoring of this group of patients has shown a high incidence of ventricular arrhythmias (multiform, couplets, ventricular tachycardia (VT), and ventricular fibrillation (VF)). ${ }^{4}$ Computer-assisted monitoring systems are suggested for arrhythmia detection in the intensive care setting because of their high detection rate. ${ }^{5,6}$ Lown and Wolf introduced a grading system of ventricular extrasystoles ${ }^{1}$ and this has been followed as the basis for treatment of ventricular arrhythmias in the acute coronary care setting. Lidocaine has been established as the drug of choice for the acute management of ventricular arrhythmias ${ }^{7}$ and has been recommended for prophylaxis of ventricular fibrillation in the presence of an acute myocardial infarction. ${ }^{8-10}$

Few studies have shown that lidocaine reduces the frequency of ventricular arrhythmias in postoperative CABG patients. Fall and his group were able to reduce 
significantly the incidence of ventricular fibrillation immediately after the aortic cross-clamp was removed by using a lidocaine infusion. "Morganroth and his group (in a double-blind parallel study comparing tocainide and lidocaine) showed a 71 per cent favourable response in the treatment of ventricular arrhythmias with lidocaine. This study included all types of postoperative cardiac surgical procedures including CABG, valve replacement, and CABG plus valve replacement. ${ }^{12}$

Using a double-blind, prospective, randomized trial, we designed a study to determine the frequency of ventricular arrhythmias and whether lidocaine administered intravenously from the time of coming off cardiopulmonary bypass and through the next $24 \mathrm{hr}$ would decrease or prevent important ventricular arrhythmias in the postoperative period in those patients who had undergone $\mathrm{CABG}$.

\section{Method}

The study was approved by the Human Investigation Committee of the Faculty of Medicine, Memorial University of Newfoundland. Informed, written consent was obtained from each patient before entry into the study. Patients scheduled for coronary artery bypass surgery were eligible for the study. Patients were excluded preoperatively if they were receiving medication for control of ventricular arrhythmias, surgery was to include valve replacement or resection of ventricular aneurysm, or the patient was allergic to lidocaine. Patients consenting to the study were further excluded from the study, intraoperatively, if lidocaine was required before coming off cardiopulmonary bypass; or if upon reperfusion of the heart, VF or VT occurred requiring defibrillation and/or lidocaine.

Patients were randomly assigned a computer-generated number to receive either placebo (normal saline) or lidocaine (Xylocaine $\left.{ }^{(}\right)$. ${ }^{*}$ All solutions were prepared by our pharmacy department. The solutions were allocated using a double-blind, placebo-controlled technique so neither attending staff nor patients knew which solution was being used.

Data collected on each patient preoperatively included: demographic information (age, gender, height, weight), New York Heart Association (NYHA) functional status, medication, degree of coronary artery stenosis, previous CABG, previous myocardial infarction, serum electrolytes, haematology, liver function, renal function, 12lead ECG.

All patients received premedication with either 0.25 to $0.75 \mathrm{mg}$ triazolam $\mathrm{PO}$ or a combination of pantopon and hyoscine IM. All patients received their usual doses of cardiac medications, and $2.5 \mathrm{~cm}$ of nitropaste, one hour preoperatively. A large-bore peripheral IV and an intraarterial line were placed using local anaesthesia. The induction sequence included $50-75 \mu \mathrm{g} \cdot \mathrm{kg}^{-1}$, fentanyl given IV over a three- to five-minute period along with an intubating dose of $0.15 \mathrm{mg} \cdot \mathrm{kg}^{-1}$ of pancuronium. Anaesthesia was supplemented with further increments of fentanyl, diazepam, and/or isoflurane or enflurane. A thermal dilution pulmonary artery catheter was placed via the right internal jugular vein, after induction of anaesthesia.

Coronary artery bypass grafts were performed using vein grafts and internal mammary artery in selected cases. Cardiopulmonary bypass (CPB) was carried out at a temperature of $25-28^{\circ} \mathrm{C}$. Patients were weaned from $\mathrm{CPB}$ when body temperature was above $35^{\circ} \mathrm{C}$ and serum potassium was less than $6 \mathrm{mmol} \cdot \mathrm{L}^{-1}$. Intraoperative blood gas analysis, aortic cross-clamp time, cardiopulmonary bypass time, temperature, and number of grafts were recorded.

Upon successful completion of CABG and removal from cardiopulmonary bypass each study patient received a $5 \mathrm{ml}$ solution of placebo or lidocaine $100 \mathrm{mg} \mathrm{IV}$, followed by an infusion of $6 \mathrm{ml} \cdot \mathrm{hr}^{-1}$ of placebo or lidocaine (equivalent to $2 \mathrm{mg} \cdot \mathrm{min}^{-1}$ ). These patients were monitored postoperatively in the intensive care unit (ICU). Their tracheas remained intubated and their lungs were ventilated for the next 12 to $20 \mathrm{hr}$. All patients had epicardial pacing wires. Their pacing threshold was tested on arrival in ICU. Intravenous morphine and diazepam were used for sedation as required. Intravenous nitroglycerin was maintained at a rate of $0.5-1.5 \mu \mathrm{g} \cdot \mathrm{kg}^{-1} \cdot \mathrm{min}^{-1}$. Haemoglobin, electrolytes, arterial blood gas analysis, temperature, and coagulation were monitored on a regular basis. Intravenous potassium chloride was given as required to keep the serum potassium concentration above $4.0 \mathrm{mmol} \cdot \mathrm{L}^{-1}$, packed $R B C$ 's were given to maintain a haemoglobin concentration of $90-100 \mathrm{~g} \cdot \mathrm{L}^{-1}$, ventilation was adjusted to maintain normal blood gas evaluation, and inotropes or vasodilators were used as required. All patients received propranolol $10 \mathrm{mg}$ via a nasogastric tube every six hours.

The ECG was monitored continuously using Marquette ${ }^{1}$ monitors, series $7010 .{ }^{*}$ These monitors are capable of arrhythmia detection with automated alarms, printout, and electronic storage of these events. The ventricular arrhythmias were confirmed or diagnosed by the attending nurse. Hard copies of the arrhythmias were reviewed by the attending medical staff. A 12-lead ECG was obtained on arrival in ICU, and at 12 and $24 \mathrm{hr}$. The CPK-MB enzymes were assessed in patients with new 
postoperative ECG changes on either of the 12-lead ECGs or when clinically indicated. New Q-waves on the 12-lead ECG of more than $0.04 \mathrm{sec}$ in duration and $1 \mathrm{~mm}$ or more in depth, and a positive CPK-MB was considered diagnostic of a postoperative myocardial infarction. New ST changes were considered significant if greater than $1 \mathrm{~mm}$ of ST-depression was present, as measured $0.06 \mathrm{sec}$ from the J point, or new T-wave inversion occurred.

The outcome variable in this trial was the presence or absence of clinically significant premature ventricular contractions (PVC):

$1>5$ PVC's per minute,

2 Multifocal PVC's, bigeminy,

3 Couplets,

4 Ventricular tachycardia or R on T,

5 Ventricular fibrillation.

In the event that any of these ventricular arrhythmias occurred, the patient received the appropriate clinical treatment for that arrythmia. The study code was broken at that time only.

\section{Statistical method}

The descriptive, laboratory, clinical, and surgical data were compared to monitor the similarity of the two randomly assigned groups of patients. The development of ventricular arrhythmias in the first $24 \mathrm{hr}$ post-surgery was the main outcome variable. Other unfavourable outcomes (Table IV) were also examined to ensure that treatment did not lead to an increase in other problems.

The frequency of ventricular arrhythmias in the lidocaine and placebo groups was tested by chi-square analysis with Yates' correction at $P<0.05$. The number of subjects was selected to detect a 50 per cent decrease in frequency of arrhythmias with 70 per cent assurance (Type Il error $=0.03$ ) at 95 per cent confidence (Type I error $=0.05$ ) from an expected arrhythmia frequency of 67 per cent in the control group (Kraemer and Thieman 1987). ${ }^{13}$ The other outcomes were tested at $P<0.10$ to ensure that other unfavourable outcomes did not increase with active treatment.

\section{Results}

A total of 120 CABG patients consented to enter the study. Of these, $37 \mathrm{had}$ ventricular fibrillation or ventricular tachycardia on reperfusion of the heart at the time of release of the aortic cross-clamp or before coming off bypass, or had received lidocaine for ventricular arrhythmias before coming off bypass. These patients were excluded from the study. Of the remaining 83 patients, 40 had been randomly assigned to the lidocaine group and 43 to the placebo group.

Table I is an outline of the demographic information and preoperative clinical status of each group. They were
TABLE I Clinical status of subjects before surgery

\begin{tabular}{|c|c|c|c|}
\hline \multirow[b]{2}{*}{ Variable } & \multicolumn{2}{|l|}{ Group } & \multirow{2}{*}{$\begin{array}{l}\text { Pooled } \\
\text { standard } \\
\text { deviarion }\end{array}$} \\
\hline & Lidocaine & Placebo & \\
\hline$n$ & 40 & 43 & \\
\hline Age $(y r)$ & 57 & 60 & \pm 8.5 \\
\hline Minimum & 44 & 38 & \\
\hline Maximum & 79 & 78 & \\
\hline Sex $M$ & 37 & 36 & \\
\hline F & 3 & 7 & \\
\hline Ratio & 12.3 & 5.1 & \\
\hline Height (cm) & 169 & 168 & \pm 9.3 \\
\hline Weight (kg) & 80.5 & 81.8 & \pm 12.08 \\
\hline Temp. ${ }^{\circ} \mathrm{C}$ & 36.5 & 36.5 & \pm 0.3 \\
\hline Heart Rate $\cdot \mathrm{min}^{-1}$ & 65 & 69 & \pm 9.7 \\
\hline Systolic BP mmHg & 120 & 122 & \pm 13.3 \\
\hline Diastolic BP mmHg & 74 & 74 & \pm 7.7 \\
\hline \multicolumn{4}{|l|}{ Medication } \\
\hline Beta blocker & 5 & 7 & \\
\hline Calcium blocker & 13 & 15 & \\
\hline Both beta + Ca & 20 & 20 & \\
\hline Neither & 2 & 1 & \\
\hline \multicolumn{4}{|l|}{ NYHA status } \\
\hline 1 & 1 & 2 & \\
\hline 2 & 8 & 5 & \\
\hline 3 & 19 & 23 & \\
\hline 4 & 12 & 13 & \\
\hline \multicolumn{4}{|l|}{ Vessel disease } \\
\hline triple or more & 31 & 33 & \\
\hline double & 8 & 8 & \\
\hline single & 1 & 2 & \\
\hline Left main $>70 \%$ stenosis & 7 & 6 & \\
\hline Previous myocardial infarction & 18 & 24 & \\
\hline Ptevious CABG & 4 & 5 & \\
\hline
\end{tabular}

comparable in age, sex, height, and weight. This includes heart rate, blood pressure, left main coronary artery stenosis ( $>70$ per cent stenosis), previous myocardial infarction, and previous CABG. Preoperative blood biochemistry (including serum electrolytes, BUN, creatinine, prothrombin time, albumin, haemaglobin, and platelets) was similar for both groups. The pump duration, aortic cross-clamp time, serum potassium off bypass, and use of internal mammary artery graft is described for each group in Table II.

TABLE II Variables during surgery

\begin{tabular}{lccc}
\hline Variables & Lidocaine & Placebo & Pooled SD \\
\hline$n$ & 40 & 43 & \\
Pump duration (min) & 104.4 & 100.6 & 27.01 \\
X-clamp time (min) & 65.8 & 64.5 & 16.42 \\
Potassium $\left(\mathrm{mmol} \cdot \mathrm{L}^{-1}\right)$ & 5.2 & 5.2 & 0.93 \\
Internal mammary & 18 & 21 & \\
\hline
\end{tabular}

X-clamp - aortic cross-clamp. 
TABLE III Post-surgery outcome of patients in the intensive care unit

\begin{tabular}{llc}
\hline Variable & Lidocaine & Placebo \\
\hline Number & 40 & 43 \\
Arthythmias: & 0 & 2 \\
$\quad$ VF & 6 & 13 \\
VT & 3 & 4 \\
Couplets & 1 & 5 \\
$\quad$ Bigeminy & 3 & 5 \\
PVCS & 13 & 29 \\
Total & 27 & 14 \\
No arrhythmias & &
\end{tabular}

Test for all arrhythmias: $\chi^{2}=8.67, P<0.005$.

Test for VT + VF arrhythmias: $\chi^{2}=3.30, P<0.01$.

VF - ventricular fibrillation.

VT - ventricular tachycardia.

PVCS - premature ventricular contractions.

Table III shows the type and frequency of ventricular arrhythmias for each group. No patient in the lidocaine group developed ventricular fibrillation while two patients in the placebo group had VF requiring CPR and defibrillation.

Ventricular fibrillation occurred in one male patient in the placebo group, at the time of testing the epicardial pacemaker. The VF was preceded by VT. The pre- and post-bypass ECGs were unchanged from the preoperative ECG. Blood gas analyses and electrolytes were normal. In the second patient receiving placebo, VF occurred early in the postoperative period. This patient had undergone a previous CABG three years before this study. His preoperative coronary angiogram showed blocked grafts, an occluded right coronary artery, and 90 per cent stenosis of the left anterior descending coronary artery. Ventricular fibrillation was immediately preceded by bigeminy and VT. Blood gas analyses and electrolytes were normal at the time. The ECG showed new anterolateral ST depression.

Thirteen patients in the placebo group had ventricular tachycardia compared with six in the lidocaine group. The distribution of couplets, bigeminy, and PVCs $>5 \cdot \mathrm{min}^{-1}$ are displayed in Table III. Overall, there was a significant reduction of ventricular arrhythmias $(P<0.005)$ in the lidocaine group ( 33 per cent) versus the placebo group (67 per cent). More importantly, there was a significant reduction in ventricular tachycardia and ventricular fibrillation in the lidocaine group.

There was no apparent increase in adverse effects of lidocaine as demonstrated by the similar numbers of new myocardial infarcts, new ST changes on ECG, use of intra-aortic balloon pump, or use of inotropic agents in both groups. There were no deaths in either group, but two cardiac arrests (VF) in the placebo group (Table IV).
TABLE IV Other clinical events during the $24 \mathrm{hr}$ after surgery

\begin{tabular}{lcc}
\hline Variable & Lidocaine & Placebo \\
\hline Myocardial infarction & 4 & 4 \\
ST changes & 18 & 23 \\
Intraaoric balloon & 2 & 3 \\
Inotropic drugs & 8 & 12 \\
Cardiac arrests & 0 & 2 \\
Deaths & 0 & 0 \\
Events among: & & \\
$\quad$ arrhythmics & 12 & $33 \dagger$ \\
non-arrhythmics & $20^{*}$ & $17 \dagger$ \\
\hline
\end{tabular}

*Somewhat less.

†Somewhat more than expected: $P<0.10, x^{2}=3.12$.

\section{Discussion}

The results of this study showed a high incidence (67 per cent) of clinically important ventricular arrhythmias in the postoperative CABG patients in the placebo control group. The incidence of postoperative ventricular arrhythmias in CABG patients has been reported at 20-36 per cent. ${ }^{4,14}$ The incidences in these two studies are lower than one would expect in an ambulatory group of patients with coronary artery disease. Lown performed 24-hr Holter monitoring on 100 outpatient males and 84 inpatients referred for coronary angiography. This group revealed a 27 per cent incidence of ventricular couplets, 14 per cent ventricular tachycardia, and 25 per cent multiform PVCs. ${ }^{2}$ The result was a 66 per cent incidence of clinically important PVCs in ambulatory patients with known coronary artery disease.

In the current study, efforts were made to exclude a subgroup of patients who were considered to be at a higher risk of developing postoperative ventricular arrhythmias. Particularly, those patients who required surgery for valve replacement; ${ }^{4,14}$ or medication for control of ventricular arrhythmias preoperatively; or defibrillation and/or antiarrhythmics for ventricular arrhythmias before coming off cardiopulmonary bypass.

The comparable group of patients given IV lidocaine prophylactically showed a significant reduction in the ventricular arrhythmia rate to 33 per cent $(P<0.005)$, compared with the placebo group of 67 per cent. Furthermore, there was significant reduction of ventricular fibrillation and ventricular tachycardia in the lidocaine treated group $(P<0.01)$.

Campbell reviewed the literature related to the treatment and prophylaxis of ventricular arrhythmias in acute myocardial infarction. ${ }^{15}$ The observed incidence of VF in hospital coronary care units was four to ten per cent with an acute myocardial infarction. The incidence of primary $\mathrm{VF}$ decreased exponentially with time and was rare after 
$12 \mathrm{hr}$. Three-beat VT was a relatively common occurrence within the first $24 \mathrm{hr}$ of an acute MI (up to 73 per cent) and was rarely of haemodynamic significance. Warning arrhythmias occurred at about the same frequency in infarct patients without VF as those who develop VF, and so had very little predictive value in determining which patient was likely to develop VF.

A number of factors other than coronary heart disease may be expected to increase the incidence of ventricular arrhythmias in postoperative CABG patients in the first 24 $\mathrm{hr}$. Recent surgical manipulation of the heart and coronary vessels may lead to a more irritable ventricle or coronary spasm. Instrumentation of the heart with a pulmonary artery catheter, epicardial pacing wires, or chest tubes may have a similar irritating effect. Metabolic and physiological derangements of serum electrolytes, arterial blood gases, inadequate oxygen delivery to the myocardium, low haemoglobin concentration, body temperature changes, instability of blood pressure and heart rate may play a role. An increased level of stress secondary to postoperative pain, irritation of the tracheal tube, invasive monitoring, and the unfamiliar ICU setting may produce excess catecholamine release.

The infarction rate of nine per cent and new ST changes in 53 per cent of patients after surgery in the control group may have contributed to the higher incidence of postoperative ventricular arrhythmias. In addition, serum lidocaine concentrations were not measured in the treated group. Lidocaine was given as an initial bolus of $100 \mathrm{mg}$ followed by an infusion of $2 \mathrm{mg} \cdot \mathrm{min}^{-1}$. Serum levels may not have reached the therapeutic range of $2-5 \mu \mathrm{g} \cdot \mathrm{ml}^{-1}$ in the first one or two hours. ${ }^{5}$

This double-blind controlled study has shown a 67 per cent incidence of ventricular arrhythmias in the postoperative CABG patients in the control group. This arrythmia rate was reduced to 33 per cent by using intravenous prophylactic lidocaine throughout the first $24 \mathrm{hr}$ period after surgery. Many of the ventricular arrhythmias in the lidocaine group were controlled by an additional bolus of 50-100 $\mathrm{mg}$ of lidocaine and by increasing the infusion rate to $3 \mathrm{mg} \cdot \mathrm{min}^{-1}$. This suggests that there may be a role for the use of prophylactic IV lidocaine in the postoperative CABG patient to reduce the incidence of ventricular arrhythmias. The implications for defibrillation and cardiopulmonary resuscitation in the early postoperative cardiac patient may be considerably different than for the nonsurgical patient. The recent sternotomy may become contaminated, infected, or disrupted. There is increased risk of bleeding or disruption of the new coronary grafts and of myocardial damage with defibrillation. There also is the danger of dislodging chest tubes, tracheal tube, or invasive monitoring equipment. Therefore, it is recommended that a routine infusion of lidocaine be given to every postoperative coronary bypass patient for at least the first 24 hours.

\section{Acknowledgements}

Dr. D.G. Bryant, biostatistician, in the Faculty of Medicine; Department of Pharmacy, HSC; staff and residents.

\section{References}

1 Lown B, Wolf $M$. Approaches to sudden death from coronary heart disease. Circulation 1971; 54: 130-40.

2 Lown B, Calvert AF, Armington $R$, Ryan $M$. Monitoring for serious arrhythmias and high risk of sudden death. Circulation 1975; 51 (Suppl III): 189-98.

3 Bigger JT, Weld FM. Analysis of prognostic significance of ventricular arrhythmias after myocardial infarction. Br Heart J 1981; 45: 717-24.

4 Dewar ML, Rosengarten MD, Blundell PE, Chu-Jeng Chiu $R$. Perioperative Holter monitoring and computer analysis of dysrhythmias in cardiac surgery. Chest 1985; 87 : 593-7.

5 Vetter NJ, Julian DG. Comparison of arrhythmias computer and conventional monitoring in coronary-care unit. Lancet 1975; 1151-54.

6 Alcover IA, Henning RJ, Jackson DL. A computerassisted monitoring system for arthythmias detection in a medical intensive care unit. Crit Care Med 1984; 12: 888-91.

7 Gianelly $R$, von der Groeben JO, Spivack AP, Harrison $D C$. Effect of lidocaine on ventricular arrhythmias in patients with coronary heart disease. N Engl J Med 1967; 277: 1215-9.

8 Lie KI, Wellens HJ, van Capelle FJ, Durrer D. Lidocaine in the prevention of primary ventricular fibrillation. $N$ Engl J Med 1974; 291: 1324-6.

9 DeSilva RA, Lown B, Hennekens $C H$, Casscells $W$. Lignocaine prophylaxis in acute myocardial infarction: an evaluation of randomised trials. Lancet 1981; 2: 855-8.

10 Koster $R W$, Dunning $A J$. Intramuscular lidocaine for prevention of lethal arrhythmias in the prehospitalization phase of acute myocardial infarction. $N$ Engl J Med 1985; 313: 1105-10.

11 Fall SM, Burton NA, Graeber GM et al. Prevention of ventricular fibrillation after myocardial revascularization. Ann Thorac Surg 1987; 43: 182-4.

12 Morganroth J, Pandis IP, Harley $S$ et al. Efficacy and safety of intravenous tocainide compared with intravenous lidocaine for acute ventricular arrhythmias immediatcly after cardiac surgery. Am J Cardiol 1984; 54: 1253-8.

13 Kraemer $H C$, Thiemann $S$. How many subjects?: statistical power and analysis in research. Newbury Park: Sage Publications, 1987. 
14 Michelson EL, Morganroth J, MacVaugh H. Postoperative arrhythmias after coronary artery and cardiac valvular surgery detected by long-term electrocardiographic monitoring. Am Heart J 1979; 97: 442-8.

15 Campbell $R W F$. Treatment and prophylaxis of ventricular arrhythmias in acute myocardial infarction. Am J

Cardiol 1983; 52: 55C-59C. 